C2018 IEEE. Personal use of this material is permitted. Permission from IEEE must be obtained for all other uses, in any current or future media, including reprinting/republishing this material for advertising or promotional purposes, creating new collective works, for resale or redistribution to servers or lists, or reuse of any copyrighted component of this work in other works. This is the author's version of an article that has been published in the conference proceedings. The final version of record is available at https://doi.org/10.1109/PLANS.2018.8373480 


\title{
Exchanging Transmitter Maps in Multipath Assisted Positioning
}

\author{
Markus Ulmschneider*, David Calvo Luz ${ }^{\dagger}$, Christian Gentner* \\ ${ }^{*}$ German Aerospace Center (DLR), Institute of Communications and Navigation \\ Münchner Str. 20, 82234 Wessling, Germany \\ Email: \{markus.ulmschneider, christian.gentner\}@dlr.de \\ ${ }^{\dagger}$ Polytechnic University of Madrid (UPM), Escuela Técnica Superior de Ingenieros de Telecomunicación (ETSIT) \\ Avenida Complutense 30, 28040 Madrid, Spain \\ Email: david.calvoluz@gmail.com
}

\begin{abstract}
While multipath propagation has commonly been regarded as a drawback for wireless localization technologies, the spatial information contained in multipath components (MPCs) can be exploited for positioning a user. In multipath assisted positioning, each MPC arriving at a receiver is regarded as a line-ofsight signal from a virtual transmitter. We assume the locations of the physical and virtual transmitters to be unknown and estimate them jointly with the user position with simultaneous localization and mapping (SLAM). In a setting where multiple users move in the same scenario, maps of physical and virtual transmitters can be exchanged among them. However, these maps are in different local coordinate systems with unknown relative rotation and translation. The distances among transmitters within each map are exploited in order to find correspondences among transmitters in different maps. Based on the correspondences, the unknown rotation and translation parameters are estimated. This allows a user to exploit the information in a transmitter map received from other users, and hence extends our multipath assisted positioning approach from a single user to a cooperative radiolocation algorithm. In simulations in an indoor scenario we show that using a prior transmitter map decreases the user positioning error although the map is in an unknown coordinate system different from the user's.
\end{abstract}

\section{INTRODUCTION}

The positioning performance of global navigation satellite systems (GNSSs) is sufficient for many applications in scenarios with a clear view to the sky. In environments like indoors or in urban canyons, effects such as multipath propagation, a low received signal power or signal blockage decrease the positioning performance of GNSSs drastically, and the precise localization of a user remains a challenge [1]. Nevertheless, other radio frequency (RF) signals are often available in such scenarios, and they can be used as signals of opportunity (SoOs) for localization. For example, cellular signals are available in nearly all populated areas. Hence, for localization using cellular signals, no additional infrastructure for transmission or reception has to be installed.

Positioning approaches using SoOs suffer from multipath propagation as well when standard methods to combat multipath propagation are used. In particular in scenarios such as urban canyons or indoors, a high multipath propagation can be expected. Instead of trying to combat multipath propagation, the spatial information from multipath components (MPCs) can be exploited. Such an approach is called multipath assisted positioning. In multipath assisted positioning, each MPC is regarded as a signal transmitted by a virtual transmitter in a line-of-sight (LoS) condition. These virtual transmitters can be used for localizing a user.

Some approaches in multipath assisted positioning assume the geometry of the environment, for example as a floorplan, and the physical transmitter locations to be known in advance [2], [3]. Based on this information, the locations of the virtual transmitters can be calculated. Our approach does not rely on such prior knowledge. Hence, the locations of both the physical and the virtual transmitters are unknown. Instead, in our approach named Channel-SLAM [4], we estimate the locations of the physical and virtual transmitters jointly with the user position in a simultaneous localization and mapping (SLAM) scheme.

In many GNSS denied environments such as in urban canyons or public buildings, a high fluctuation of users can be expected. In other words, many users move through a scenario on the same or on different trajectories. These users can cooperate by exchanging maps of observed physical and virtual transmitters either directly, or via some local entity. Such an entity could be a base station in a cellular network. A user entering a scenario can use a map of transmitters from one or multiple previous users as prior knowledge on the transmitter locations. We call such a map a prior map. A map estimated by a user is called a user map. Each map consists of a set of transmitters whose states are represented by probability density functions (PDFs).

However, since Channel-SLAM is a relative localization approach, the user map and a prior map are in different coordinate systems with an unknown rotation and an unknown translation. In addition, the correspondences among the transmitters in the two maps are not known. Hence, we define finding a match between the two maps as both (1) estimating the relative rotation and translation between the two coordinate systems, and (2) finding correspondences among transmitters in the two maps. Only when a reliable match between user 


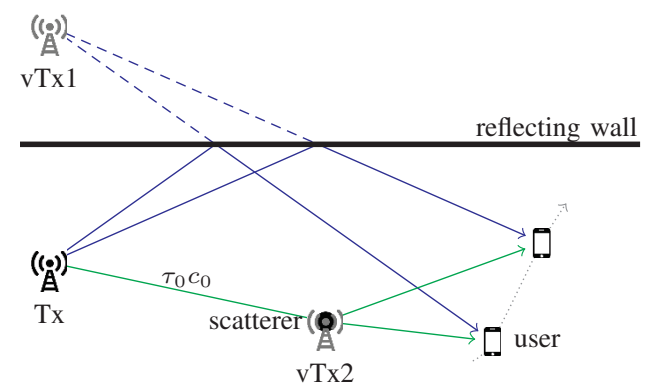

Fig. 1. The signal transmitted by the physical transmitter Tx is received by the user via two different propagation paths. The blue signal component is reflected at the wall and regarded as being transmitted by the virtual transmitter vTx 1 . The green signal component is scattered by a point scatterer and regarded as being transmitted by the virtual transmitter vTx2, which is located at the scatterer location.

and prior map is found, the information in the prior map can be exploited by the user.

The remainder of the paper is structured as follows. Section $\Pi$ introduces the idea of multipath assisted positioning and Channel-SLAM. In Section III we derive how to match two maps. We evaluate our algorithm based on simulations in an indoor scenario in Section IV] Finally, Section $\mathrm{V}$ concludes the paper.

\section{PRINCIPLES}

\section{A. Multipath Assisted Positioning}

The idea of multipath assisted positioning and virtual transmitters is illustrated in Fig. 11 The physical transmitter Tx radiates an RF signal. The signal component in dark blue is reflected at the wall and arrives at the user as a MPC. Though, this signal component is regarded as transmitted by the virtual transmitter vTx1 in a LoS condition. As the user moves, the reflection point of the signal at the wall moves as well, but the location of vTx1 is static. In particular, the location of vTx 1 is the location of the physical transmitter mirrored at the reflecting wall. The physical transmitter Tx and the virtual transmitter vTx 1 are inherently perfectly time synchronized.

The signal component in green arrives at the user after being scattered by a point scatterer. It is regarded as a LoS signal from the virtual transmitter vTx2, which is located at the position of the point scatterer. Again, the virtual transmitter location is static as the user is in motion. Though, the transmitters Tx and vTx2 are not time synchronized when the signal is scattered. The virtual transmitter vTx2 has a delay offset $\tau_{0}$ towards the physical transmitter Tx, which is the Euclidean distance between the two transmitters divided by the speed of light $c_{0}$. The delay offset $\tau_{0}$ can be interpreted as a clock offset.

A generalization to the case where the transmitted signal is reflected and/or scattered multiple times is straightforward [4].

\section{B. Channel-SLAM}

We assume a linear, time-variant multipath channel between a static physical transmitter and a mobile user. The signal arriving at the user's RF receiver is therefore a superposition of signal components, each with its own power, phase, time of arrival (ToA) and angle of arrival (AoA).

The Channel-SLAM algorithm works in two stages: In a first stage, the Kalman enhanced super resolution tracking (KEST) estimator [5] is used to estimate the parameters of the signal components arriving at the receiver and track them over time. Such parameters can be the complex amplitude, the ToA or the AoA, for example. In a second stage, the KEST estimates are used as measurements to track the position of the user and estimate the locations of physical and virtual transmitters with SLAM. Again, each signal component arriving at the receiver corresponds to a physical or virtual transmitter whose location is not known. Since Channel-SLAM does not differentiate between physical and virtual transmitters, i.e., between the LoS component and MPCs, the term transmitter is used as a general term referring to either of them in the following.

The ToA and AoA estimates from the KEST algorithm at time instant $k$ are stacked in the measurement vector

$$
\boldsymbol{z}_{k}=\left[\begin{array}{llllll}
d_{1, k} & \ldots & d_{N_{\mathrm{TX}}, k} & \theta_{1, k} & \ldots & \theta_{N_{\mathrm{TX}}, k}
\end{array}\right]^{T},
$$

where $d_{j, k}$ denotes the ToA and $\theta_{j, k}$ the AoA of the $j^{\text {th }}$ transmitter. The number of observable transmitters is denoted by $N_{\mathrm{TX}}$. Although the number of transmitters might change over time, the time index in $N_{\mathrm{TX}}$ is omitted for the sake of notational brevity.

In the second stage of Channel-SLAM, the estimates from Eq. (1) are used to estimate the user and transmitter locations. In SLAM terms, we localize the user and map the transmitters simultaneously. The combined state vector at time instant $k$ consists of the user state $\boldsymbol{x}_{\mathrm{u}, k}$ and the states of the $N_{\mathrm{TX}}$ transmitters,

$$
\boldsymbol{x}_{k}=\left[\begin{array}{llll}
\boldsymbol{x}_{\mathrm{u}, k}^{T} & \boldsymbol{x}_{\mathrm{TX}, k}^{<1>T} & \ldots & \boldsymbol{x}_{\mathrm{TX}, k}^{<N_{\mathrm{TX}}>^{T}}
\end{array}\right]^{T},
$$

where $\boldsymbol{x}_{\mathrm{TX}, k}^{<j>}$ denotes the state of the $j^{\text {th }}$ transmitter. The user state includes the position and velocity in two dimensions, i.e.,

$$
\boldsymbol{x}_{\mathrm{u}, k}=\left[\begin{array}{llll}
x_{k} & y_{k} & v_{x, k} & v_{y, k}
\end{array}\right]^{T} .
$$

Since transmitters are assumed static, the state of the $j^{\text {th }}$ transmitter consists of its location and clock offset $\tau_{0, k}^{<j>}$, namely

$$
\boldsymbol{x}_{\mathrm{TX}, k}^{<j>}=\left[\begin{array}{lll}
x_{\mathrm{TX}, k}^{<j>} & y_{\mathrm{TX}, k}^{<j>} & \tau_{0, k}^{<j>}
\end{array}\right]^{T} .
$$

We apply recursive Bayesian estimation [6] to estimate the posterior PDF $\mathrm{p}\left(\boldsymbol{x}_{0: k} \mid \boldsymbol{z}_{1: k}\right)$, where $\boldsymbol{x}_{0: k}$ denotes the state vector from time instants 0 to $k$, and $z_{1: k}$ the measurements from time instants 1 to $k$. The posterior can be factorized into

$$
\begin{aligned}
\mathrm{p}\left(\boldsymbol{x}_{0: k} \mid \boldsymbol{z}_{1: k}\right) & =\mathrm{p}\left(\boldsymbol{x}_{\mathrm{TX}, 0: k}, \boldsymbol{x}_{\mathrm{u}, 0: k} \mid \boldsymbol{z}_{1: k}\right) \\
& =\mathrm{p}\left(\boldsymbol{x}_{\mathrm{u}, 0: k} \mid \boldsymbol{z}_{1: k}\right) \mathrm{p}\left(\boldsymbol{x}_{\mathrm{TX}, 0: k} \mid \boldsymbol{x}_{\mathrm{u}, 0: k}, \boldsymbol{z}_{1: k}\right) \\
& =\mathrm{p}\left(\boldsymbol{x}_{\mathrm{u}, 0: k} \mid \boldsymbol{z}_{1: k}\right) \prod_{j=1}^{N_{\mathrm{TX}}} \mathrm{p}\left(\boldsymbol{x}_{\mathrm{TX}, 0: k}^{<j>} \mid \boldsymbol{x}_{\mathrm{u}, 0: k}, \boldsymbol{z}_{1: k}\right) .
\end{aligned}
$$


In the last step in Eq. (5), we assume independence among the measurements for different transmitters, i.e., for different signal components. The actual estimation is performed by a Rao-Blackwellized particle filter [4], [7]. For every user particle, the state of each transmitter is estimated independently from the other transmitters by an own particle filter. In the user particle filter, the user posterior state PDF from Eq. (5) is approximated by

$$
\mathrm{p}\left(\boldsymbol{x}_{\mathrm{u}, k} \mid \boldsymbol{z}_{1: k}\right)=\sum_{i=1}^{N_{p}} w_{k}^{<i>} \delta\left(\boldsymbol{x}_{\mathrm{u}, k}-\boldsymbol{x}_{\mathrm{u}, k}^{<i>}\right),
$$

where $\boldsymbol{x}_{\mathrm{u}, k}^{<i>}$ is the $i^{\text {th }}$ user particle, $w_{k}^{<i>}$ its associated weight, $N_{p}$ the number of user particles, and $\delta(\cdot)$ the Dirac distribution. Likewise, the posterior state PDF of $\boldsymbol{x}_{\mathrm{TX}, k}^{<i, j>}$, i.e., the $j^{\text {th }}$ transmitter for the $i^{\text {th }}$ user particle, is represented as

$\mathrm{p}\left(\boldsymbol{x}_{\mathrm{TX}, k}^{<i, j>} \mid \boldsymbol{z}_{1: k}, \boldsymbol{x}_{\mathrm{u}, k}^{<i>}\right)=\sum_{l=1}^{N_{p, \mathrm{Tx}}} w_{k}^{<i, j, l>} \delta\left(\boldsymbol{x}_{\mathrm{TX}, k}^{<i, j>}-\boldsymbol{x}_{\mathrm{TX}, k}^{<i, j, l>}\right)$,

where $\boldsymbol{x}_{\mathrm{TX}, k}^{<i, j, l>}$ is the $l^{\text {th }}$ particle, $w_{k}^{<i, j, l>}$ its associated weight, and $N_{p, \text { Tx }}$ the number of particles for that transmitter. A full derivation of Channel-SLAM can be found in [4].

\section{ESTIMATION OF ROTATION AND TRANSLATION PARAMETERS AMONG TWO MAPS}

As the user travels through a scenario, the information contained in a prior map obtained from some entity can not be used until a reliable map match between the user map and the prior map is found, i.e., until the rotation and translation parameters between the coordinate systems are estimated. Therefore, we try to find a match between the two maps at each time instant $k$ as described below. Once a reliable match is found, the transmitters in the prior map are used as prior information when the user initializes new transmitters.

Our approach to find a match between two maps is to first find correspondences among the transmitters in the two maps and subsequently the corresponding rotation and translation of the coordinate systems. We assume no dilation or skew between the coordinate systems.

Each measurement of a signal component, or transmitter, is two-dimensional, assuming ToA and AoA measurements, whereas a transmitter's state is of three dimensions, comprising its two-dimensional location and its clock offset. Hence, when initializing a new transmitter, the uncertainty about its state tends to be high, i.e., the variance in the state PDF of the newly initialized transmitter is high. It only decreases when the user moves through the scenario taking measurements from different locations. Thus, depending on the user trajectory, the shapes of the estimated state PDFs of one transmitter for two different users may differ considerably, for example due to different geometrical delusions of precision (GDoPs) or time spans during which the corresponding signal component can be tracked. Consequently, calculating a distance between two transmitter state PDFs estimated by different users with standard metrics or divergences such as the Kullback-Leibler divergence (KLD) might cause misleading results.

Therefore, we regard only a subset of transmitters in the user map and the prior map. In particular, we consider only those transmitters whose state PDF variances are smaller than a threshold $\delta_{\sigma}$. The distance between two transmitter state PDFs is then defined by the Euclidean distance between their means, i.e., the distance $d_{A, B}$ between two transmitters $A$ and $B$ is calculated as

$$
\begin{aligned}
d_{A, B}= & \| \sum_{i=1}^{N_{p}} \sum_{l=1}^{N_{p, \mathrm{Tx}}} w_{k}^{<i>} w_{k}^{<i, A, l>} \boldsymbol{x}_{\mathrm{TX}, k}^{<i, A, l>} \\
& -\sum_{i=1}^{N_{p}} \sum_{l=1}^{N_{p, \mathrm{Tx}}} w_{k}^{<i>} w_{k}^{<i, B, l>} \boldsymbol{x}_{\mathrm{TX}, k}^{<i, B, l>} \|,
\end{aligned}
$$

where $\|\cdot\|$ denotes the Euclidean norm of a vector.

\section{A. Identification of Transmitter Correspondences}

The set $\mathcal{U}$ of size $N_{\mathcal{U}}$ denotes the set of transmitters in the user map whose state PDF estimates have a variance smaller than $\delta_{\sigma}$, and the set $\mathcal{P}$ of size $N_{\mathcal{P}}$ denotes the corresponding set of transmitters in the prior map. In the following, we assume that $N_{\mathcal{U}} \leq N_{\mathcal{P}}$ to simplify the notation, but to drop this assumption is straightforward.

In order to reduce the computational complexity, we try to find a map match based on any $N_{T}$ transmitters in each map. The factorial of an integer $N$ is denoted by $N$ !. Since there are $\frac{N !}{N_{T} !\left(N-N_{T}\right) !}$ possibilities to choose $N_{T}$ out of $N$ transmitters, and there are $N_{T}$ ! possibilities to arrange $N_{T}$ transmitters, there is a total of

$$
N_{\mathcal{C}}=\frac{N_{\mathcal{U}} !}{N_{T} !\left(N_{\mathcal{U}}-N_{T}\right) !} \frac{N_{\mathcal{P}} !}{\left(N_{\mathcal{P}}-N_{T}\right) !}
$$

distinct sets $\mathcal{C}_{1}, \ldots, \mathcal{C}_{N_{\mathcal{C}}}$ of possible transmitter correspondences. Each set $\mathcal{C}_{u}$ contains $N_{T}$ tuples that are of the form $\left(\mathrm{U}_{q}, \mathrm{P}_{r}\right)$, describing a correspondence between the transmitter $\mathrm{U}_{q}$ from the user map and the transmitter $\mathrm{P}_{r}$ from the prior map.

At the beginning, there is no information on the relation of the transmitters in the two maps, since the maps are in different coordinate systems. However, the relative positions of the transmitters within the maps can be exploited. In particular, the relative distances between any two transmitters within each of the two maps are calculated. As mentioned above, we define the distance between two transmitters as the Euclidean distance between the means of their state PDFs. For notational brevity, we denote the distance between the transmitters $\mathrm{U}_{q}$ and $\mathrm{U}_{\tilde{q}}$ in the user map by $d_{q, \tilde{q}}^{\mathrm{U}}=d_{\mathrm{U}_{q}, \mathrm{U}_{\tilde{q}}}$ and likewise by $d_{r, \tilde{r}}^{\mathrm{P}}=d_{\mathrm{P}_{r}, \mathrm{P}_{\tilde{r}}}$ as the distance between two transmitters $\mathrm{P}_{r}$ and $\mathrm{P}_{\tilde{r}}$ from the prior map.

Fig. 22illustrates a simple example for finding a map match. There are four transmitters in light and dark blue in the user map, $\mathcal{U}=\left\{\mathrm{U}_{1}, \ldots, \mathrm{U}_{4}\right\}$, and five transmitters in light and dark green in the prior map, $\mathcal{P}=\left\{\mathrm{P}_{1}, \ldots, \mathrm{P}_{5}\right\}$. From both maps, any $N_{T}=3$ transmitters from the maps are chosen to find a match map. In Fig. 2, these are exemplarily $\mathrm{U}_{1}, \mathrm{U}_{2}$ and $\mathrm{U}_{3}$ from 


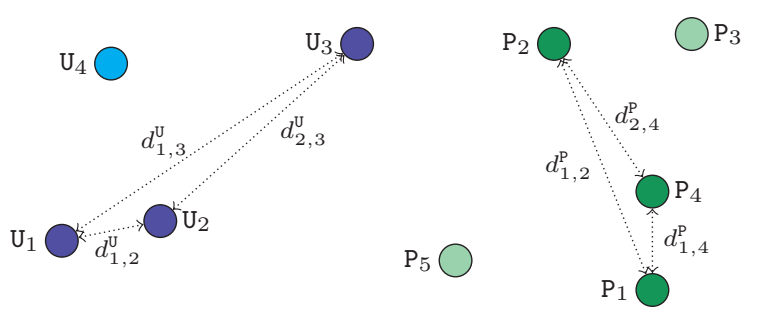

Fig. 2. The distances among the $N_{T}=3$ transmitters in light and dark blue in the user map and in light and dark green in the prior map are indicated by dotted lines. Based on the distances, correspondences among the transmitters are to be found.

the user map and $\mathrm{P}_{1}, \mathrm{P}_{2}$ and $\mathrm{P}_{4}$ from the prior map, drawn in dark blue and green, respectively. Based on the distances among the $N_{T}=3$ transmitters in each map, indicated by the dotted lines, correspondences among the transmitters are to be found.

For each possible set $\mathcal{C}_{u}$ of correspondences, the distances between any two of the $N_{T}$ regarded transmitters in the user map are compared to the distances between the corresponding two transmitters in the prior map. The squared differences between any two of these distances are summed up. The correspondence set $\hat{\mathcal{C}}$ for which this sum is minimized is the chosen set of correspondences, i.e.,

$$
\hat{\mathcal{C}}=\underset{\substack{\mathcal{C}_{u} \\ u=1, \ldots, N_{\mathcal{C}}}}{\arg \min } \sum_{\left(\mathrm{U}_{q}, \mathrm{P}_{r}\right) \in \mathcal{C}_{u} \wedge\left(\mathrm{U}_{\tilde{q}}, \mathrm{P}_{\tilde{r}}\right) \in \mathcal{C}_{u}}\left(d_{r, \tilde{r}}^{\mathrm{P}}-d_{q, \tilde{q}}^{\mathrm{U}}\right)^{2} .
$$

\section{B. Estimation of the Rotation and Translation}

Based on the $N_{T}$ correspondences of transmitters in the set $\hat{\mathcal{C}}$, the rotation $\beta$ and translation $\gamma=\left[\begin{array}{lll}\bar{x} & \bar{y} & 0\end{array}\right]$ between the coordinate systems of the user map and the prior map are estimated. First, the transmitters in the two maps are re-arranged such that the set of correspondences is $\hat{\mathcal{C}}=$ $\left\{\left(\mathrm{U}_{1}, \mathrm{P}_{1}\right), \ldots,\left(\mathrm{U}_{N_{T}}, \mathrm{P}_{N_{T}}\right)\right\}$, i.e., there is a correspondence between the $j^{\text {th }}$ transmitter in the user map and the $j^{\text {th }}$ transmitter in prior map for $j=1, \ldots, N_{T}$.

Let $\boldsymbol{\mu}_{j}^{u}=\left[\begin{array}{lll}x_{j}^{u} & y_{j}^{u} & \tau_{j}^{u}\end{array}\right]^{T}$ be the mean of the $j^{\text {th }}$ transmitter's state PDF in the user map, and accordingly $\boldsymbol{\mu}_{j}^{p}$ the mean of the $j^{\text {th }}$ transmitter's state PDF in the prior map. The means of the transmitters in the user map are combined in the matrix

$$
\boldsymbol{M}_{\mathrm{u}}=\left[\begin{array}{lll}
\boldsymbol{\mu}_{1}^{u} & \ldots & \boldsymbol{\mu}_{N_{T}}^{u}
\end{array}\right]^{T},
$$

and likewise, the matrix of the means of the transmitters in the prior map is

$$
\boldsymbol{M}_{\mathrm{p}}=\left[\begin{array}{lll}
\boldsymbol{\mu}_{1}^{p} & \ldots & \boldsymbol{\mu}_{N_{T}}^{p}
\end{array}\right]^{T} .
$$

With the rotation matrix

$$
\boldsymbol{R}_{\boldsymbol{\beta}}=\left[\begin{array}{ccc}
\cos \beta & \sin \beta & 0 \\
-\sin \beta & \cos \beta & 0 \\
0 & 0 & 1
\end{array}\right]
$$

the maps $\boldsymbol{M}_{\mathrm{p}}$ and $\boldsymbol{M}_{\mathrm{u}}$ are related by

$$
M_{\mathrm{u}}=\boldsymbol{M}_{\mathrm{p}} \boldsymbol{R}_{\boldsymbol{\beta}}+\boldsymbol{J} \gamma+\boldsymbol{E},
$$

where $\boldsymbol{J}=\left[\begin{array}{llll}1 & 1 & \ldots & 1\end{array}\right]^{T}$ is of dimension $N_{T} \times 1$, and $\boldsymbol{E}$ is a residual matrix. Let $c_{j}=\sigma_{\mathrm{U}_{j}}^{2}+\sigma_{\mathrm{P}_{j}}^{2}$, where $\sigma_{\mathrm{U}_{j}}^{2}$ and $\sigma_{\mathrm{P}_{j}}^{2}$ are the variances of the state PDFs of the $j^{\text {th }}$ transmitter in the user map and the prior map, respectively. We seek to find $\bar{x}$, $\bar{y}$ and $\beta$ that minimize the match error $\operatorname{Tr}\left(\boldsymbol{E}^{T} \boldsymbol{C}^{-1} \boldsymbol{E}\right)$, where

$$
\boldsymbol{C}=\left[\begin{array}{ccc}
c_{1} & & \mathbf{0} \\
& \ddots & \\
\mathbf{0} & & c_{N_{T}}
\end{array}\right]
$$

For minimization, $\operatorname{Tr}\left(\boldsymbol{E}^{T} \boldsymbol{C}^{-1} \boldsymbol{E}\right)$ is derived by $\beta, \bar{x}$ and $\bar{y}$, and the results are set to zero. We denote the trace of the matrix $C^{-1}$ by $T_{C}$, i.e.,

$$
T_{C}=\operatorname{Tr}\left(C^{-1}\right)=\sum_{j=1}^{N_{T}} \frac{1}{c_{j}} .
$$

Inserting the resulting equations into each other yields an estimate for the rotation parameter

$$
\hat{\beta}=\arctan (-\phi),
$$

where

$\phi=\frac{\sum_{j=1}^{N_{T}} \frac{x_{j}^{u} y_{j}^{p}}{c_{j}}-\sum_{j=1}^{N_{T}} \frac{x_{j}^{p} y_{j}^{u}}{c_{j}}-\frac{1}{T_{C}} \sum_{j=1}^{N_{T}} \frac{x_{j}^{u}}{c_{j}} \sum_{j=1}^{N_{T}} \frac{y_{j}^{p}}{c_{j}}+\frac{1}{T_{C}} \sum_{j=1}^{N_{T}} \frac{y_{j}^{u}}{c_{j}} \sum_{j=1}^{N_{T}} \frac{x_{j}^{p}}{c_{j}}}{\sum_{j=1}^{N_{T}} \frac{x_{j}^{p} x_{j}^{u}}{c_{j}}+\sum_{j=1}^{N_{T}} \frac{y_{j}^{p} y_{j}^{u}}{c_{j}}-\frac{1}{T_{C}} \sum_{j=1}^{N_{T}} \frac{x_{j}^{u}}{c_{j}} \sum_{j=1}^{N_{T}} \frac{x_{j}^{p}}{c_{j}}-\frac{1}{T_{C}} \sum_{j=1}^{N_{T}} \frac{y_{j}^{u}}{c_{j}} \sum_{j=1}^{N_{T}} \frac{y_{j}^{p}}{c_{j}}}$,

as well as translation parameters

$$
\hat{\bar{x}}=\frac{1}{T_{C}} \sum_{j=1}^{N_{T}} \frac{x_{j}^{u}}{c_{j}}-\cos (\beta) \frac{1}{T_{C}} \sum_{j=1}^{N_{T}} \frac{x_{j}^{p}}{c_{j}}+\sin (\beta) \frac{1}{T_{C}} \sum_{j=1}^{N_{T}} \frac{y_{j}^{p}}{c_{j}}
$$

and

$$
\hat{\bar{y}}=\frac{1}{T_{C}} \sum_{j=1}^{N_{T}} \frac{y_{j}^{u}}{c_{j}}-\cos (\beta) \frac{1}{T_{C}} \sum_{j=1}^{N_{T}} \frac{y_{j}^{p}}{c_{j}}-\sin (\beta) \frac{1}{T_{C}} \sum_{j=1}^{N_{T}} \frac{x_{j}^{p}}{c_{j}} .
$$

With Eq. 15, the residual matrix and consequently the match error $\operatorname{Tr}\left(\boldsymbol{E}^{T} \boldsymbol{C}^{-1} \boldsymbol{E}\right)$ can be calculated for the obtained parameters. Note that the arctan function in Eq. (18) returns values in the interval $]-\frac{\pi}{2},+\frac{\pi}{2}[$, leaving an ambiguity between the estimated angle $\hat{\beta}$ and $\hat{\beta}+\pi$. Both values have to be considered and the one with smaller match error is chosen. If the match error falls below a threshold, a reliable estimate for the parameters has been found, and the prior map is converted into the user coordinate system.

If the match error is above the threshold, no map match is performed in this time step. This may occur when the estimate for one or more transmitters is biased or has a too high variance, or if there is no actual correspondence among the transmitters in the two maps, for example due to a change in the environment. 


\section{Complexity and Implementation}

Map matching may increase the complexity of ChannelSLAM due to the high amount of possible correspondences of transmitters in the user and prior map in Eq. 107. Though, in the beginning, transmitters are initialized with a rather high variance in their state PDF. Hence, no map matching is performed until the state PDFs of at least $N_{T}$ transmitters tracked by the user have a variance smaller than the threshold $\delta_{\sigma}$. If there are exactly $N_{T}$ such transmitters, i.e., $N_{\mathcal{U}}=N_{T}$, the number of sets of possible correspondences in Eq. (10) becomes $N_{\mathcal{C}}=\frac{N_{\mathcal{P}} !}{\left(N_{\mathcal{P}}-N_{T}\right) !}$.

Since the transmitter state PDFs are expected to change only slightly during one time step, map matching may not be needed at every single time instant. Instead, it may be performed every $q^{\text {th }}$ time instant, or if the variance of a transmitter's state PDF falls below the threshold $\delta_{\sigma}$ for the first time. It can be computed in parallel to the actual ChannelSLAM algorithm.

Typically, we expect the number of transmitters in the user map to be smaller than the number of transmitters in the prior map. If in addition $2 N_{T}<N_{\mathcal{P}}, N_{\mathcal{C}}$ and hence the complexity increase with increasing $N_{T}$. On the other hand, for small $N_{T}$, ambiguities in the correspondences can arise depending on the relative geometry of the transmitters.

Once a reliable map match has been found, the transmitters from the prior map are used as prior information and incorporated in the estimation process. Data association is necessary to associate the measurements obtained by the KEST estimator with transmitters from the prior map. We incorporate the data association scheme from [8]. Every time a new signal component is detected, the corresponding new transmitter is either associated with a transmitter from the prior map, or it is initialized as a new transmitter. When the signal component is associated with a transmitter in the prior map, the initial high uncertainty, i.e., the high variance in the transmitter state PDF, can be avoided, and the user position estimate can be corrected. This increases the performance of Channel-SLAM in terms of both accuracy and computational complexity.

\section{Evaluations}

To evaluate our approach, we performed simulations in a simple indoor scenario. Fig. 3 shows a top view of a indoor mall with one physical transmitter marked by the red triangle labeled Tx. The transmitter continuously broadcasts a known RF signal with a carrier frequency of $1.5 \mathrm{GHz}$ and a bandwidth of $100 \mathrm{MHz}$. The thick black lines are walls that reflect the transmit signal, and the black dots model point scatterers.

The user walks on the trajectory of total length $346.4 \mathrm{~m}$ drawn in blue from the point labeled START to the point labeled END with a velocity of $1 \mathrm{~m} / \mathrm{s}$. Every $100 \mathrm{~ms}$, it records a snapshot of the received signal. The simulated channel impulse response (CIR) is then passed to the KEST estimator for parameter estimation. The average signal-to-noise ratio at the receiver is $3.4 \mathrm{~dB}$. Markers indicate the traveled distance every $50 \mathrm{~m}$.

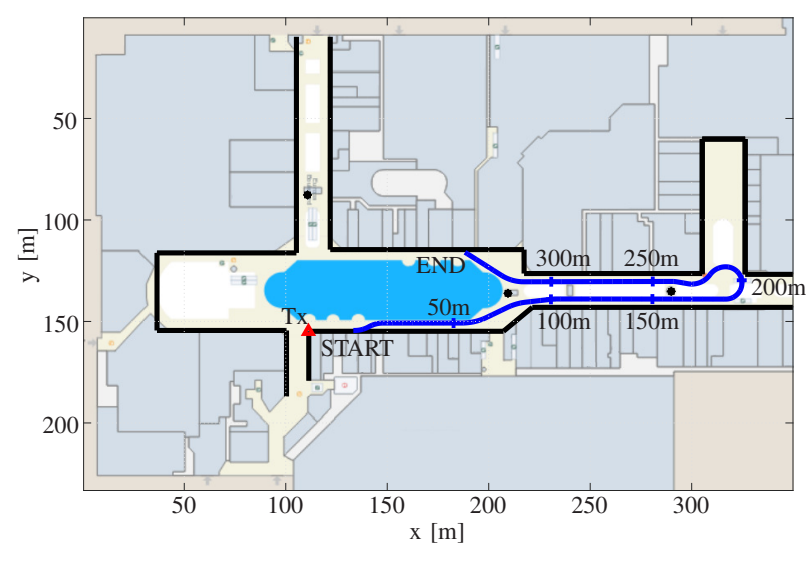

Fig. 3. Top view on the simulation scenario in an indoor mall. The physical transmitter is marked by the red triangle labeled Tx. Thick black lines are walls reflecting the transmit signal, and thick black dots point scatterers. The user walks on the blue trajectory from START to END.

The user carries an inertial measurement unit (IMU) with them. Though, only heading change rates of the IMU are used in the estimation process to avoid ambiguities of turning left or right. The user is assumed to be equipped with a two-dimensional antenna array consisting of nine elements to obtain ToA and AoA estimates from KEST.

Based on the floorplan of the indoor scenario, a prior map is created with the physical transmitter and virtual transmitters that arise due to reflections and scattering of the transmit signal. Interactions up to an order of two, i.e., single and double reflections and/or scattering, are incorporated, leading to a total of $N_{\mathcal{P}}=51$ transmitters in the prior map. Note that not all of these transmitters can be observed by the user on its trajectory. The variance of these transmitters' state PDFs is set to $2 \mathrm{~m}^{2}$ for both the $x$ and the $y$ component. Though, the prior map is in a coordinate system different from the user map, and the parameters relating the two coordinate systems are random and unknown. We set $N_{T}=4$.

Fig. 4 shows the mean absolute error (MAE) of the user versus its traveled distance averaged over 700 simulation runs. The MAE at time instant $k$ is calculated as

$$
\operatorname{MAE}_{k}=\sum_{i=1}^{N_{p}} w_{k}^{<i>}\left\|\boldsymbol{x}_{\mathrm{u}, k}^{<i>}-\dot{\boldsymbol{x}}_{\mathrm{u}, k}\right\|
$$

where $\dot{x}_{\mathrm{u}, k}$ denotes the true state of the user at time instant $k$. The red curve is the MAE when no prior map is used, while the blue curve shows the MAE with using the prior map.

As expected, both MAE curves coincide in the beginning when no match between the prior map and the user map has been found yet. The MAE without the prior map increases throughout the track. One the one hand, this is due to the geometrical delusion of precision (GDoP) getting worse as the user moves away from the physical transmitter. On the other hand, when the user moves away from the physical 


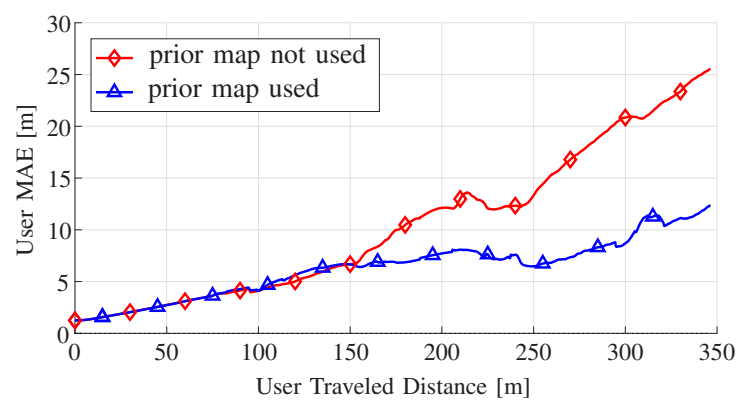

Fig. 4. The MAE of the user versus the traveled distance. No prior map is used for the curve in red, whereas a prior map is used for the curve in blue.

transmitter, less and less signal components can be tracked by KEST, and therefore less transmitters are observable and can be used for localization. When the user takes a turn and moves back towards the physical transmitter, more transmitters become observable again, but they are initialized with a high uncertainty about their state, since KEST can not associate the corresponding signal components with the previously tracked ones.

Once the estimates for the transmitters in the user map have converged far enough after a traveled distance of approximately $75 \mathrm{~m}$, a match between user and prior map can be found. From this moment on, knowledge from the prior map can be exploited by the user, and the MAE including the prior map stays considerably far below the MAE without prior map.

\section{CONCLUSION}

In multipath assisted positioning, the difficulty in exchanging transmitter maps among users is the unknown relative

\section{REFERENCES}

[1] N. Agarwal, J. Basch, P. Beckmann, P. Bharti, S. Bloebaum, S. Casadei, A. Chou, P. Enge, W. Fong, N. Hathi, W. Mann, A. Sahai, J. Stone, J. Tsitsiklis, and B. Van Roy, "Algorithms for GPS operation indoors and downtown," GPS Solutions, vol. 6, no. 3, pp. 149-160, 2002. rotation and translation between their local coordinate systems. In order to estimate the rotation and translation parameters, we first find correspondences among transmitters in the two maps. These correspondences help in a second step to actually estimate these parameters based on distances among transmitters. We have shown by simulations that using a prior transmitter map decreases the user MAE, although it is in a different and unknown coordinate system. The possibility of exchanging maps of physical and virtual transmitters among users extends Channel-SLAM from a single user to a cooperative location algorithm. In addition, if the relation of a prior map to global coordinates is known, the user position can be determined on a global scale.

\section{ACKNOWLEDGEMENT}

This work was partially supported by the EU project HIGHTS (High precision positioning for cooperative ITS applications) MG-3.5a-2014-636537 and the DLR project Navigation 4.0.

[2] P. Setlur, G. Smith, F. Ahmad, and M. Amin, "Target Localization with a Single Sensor via Multipath Exploitation," IEEE Trans. Aerosp. Electron. Syst., vol. 48, no. 3, pp. 1996-2014, Jul. 2012.

[3] P. Meissner, E. Leitinger, M. Fröhle, and K. Witrisal, "Accurate and robust indoor localization systems using ultra-wideband signals," in European Conference on Navigation (ENC), 2013.

[4] C. Gentner, T. Jost, W. Wang, S. Zhang, A. Dammann, and U.-C. Fiebig, "Multipath Assisted Positioning with Simultaneous Localization and Mapping," IEEE Trans. Wireless Commun., vol. 15, no. 9, pp. 61046117, Sep. 2016.

[5] T. Jost, W. Wang, U. Fiebig, and F. Perez-Fontan, "Detection and Tracking of Mobile Propagation Channel Paths," IEEE Trans. Antennas Propag., vol. 60 , no. 10 , pp. 4875-4883, Oct. 2012.

[6] S. Kay, Fundamentals of Statistical Signal Processing: Estimation Theory, ser. Fundamentals of Statistical Signal Processing. Prentice-Hall PTR, 1998.

[7] H. Durrant-Whyte and T. Bailey, "Simultaneous localization and mapping: part I," IEEE Robot. Autom. Mag., vol. 13, no. 2, pp. 99-110, Jun. 2006.

[8] M. Ulmschneider, C. Gentner, T. Jost, and A. Dammann, "Multiple Hypothesis Data Association for Multipath-Assisted Positioning," in 14th Workshop on Positioning, Navigation and Communications (WPNC), Oct. 2017. 